\title{
"Epizoonosis of Dermatophytosis": A Clinico - Mycological Study of Dermatophytic Infections in Central Nepal
}

\author{
Mathur M, Kedia SK, Ghimire RBK
}

Department of Dermatology, College of Medical Sciences, Bharatpur, Chitwan district, Nepal.

\section{Corresponding Author}

Mahesh Mathur

Department of Dermatology,

College of Medical Sciences, Bharatpur,

Chitwan, Nepal.

Email: maheshmathur2@hotmail.com

Citation

M. Mathur, S. K. Kedia, RBK Ghimire. "Epizoonosis of Dermatophytosis": A Clinico - Mycological Study of Dermatophytic Infections in Central Nepal. Kathmandu Univ Med J 2012;37(1):30-3.

\begin{abstract}
Background

Identification of dermatophytic species in clinical settings are important not only for epidemiological but also for the treatment.
\end{abstract}

\section{Objectives}

Present study was carried out to find out the clinical variants of Dermatophytosis and species of fungus responsible for the disease.

\section{Methods}

The prospective observational analysis of 200 clinically suspected cases of dermatophytic infection attending Dermatology department of College of Medical Sciences Teaching Hospital, Bharatpur, Chitwan, Nepal. Skin scraping, hair and nail samples were collected and processed according to standard protocol.

\section{Results}

Maximum number of patients enrolled in study were reported for treatment 5-8 weeks after the onset of disease. Overall male predominance was observed and ages between 26-30 years. Tinea corporis was the most common clinical type of tinea with female dominance in our study. $10 \%$ of cases were having extensive Tinea. $71.5 \%$ of samples were positive on direct microscopy and $62 \%$ positive on culture. Samples from T capitis were highest positive by direct microscopy (80\%) and over all dominant species of fungus isolated in our study was Trichophyton verrucosum (30.6\%).

\section{Conclusions}

The study highlighted Tinea corporis as the most common clinical type with female predominance. Overall predominant causative fungal species isolated was Trichophyton verrucosum.

\section{KEY WORD}

Dermatophyte, epidemiological factors, superficial infection,fungal species

\section{INTRODUCTION}

The prevalence of dermatophytosis varies from place to place throughout the globe and reported to be variable in different parts of same continent. In tropical and subtropical countries, it occurs in increase frequencies. ${ }^{1}$ There are about 40 species of dermatophytes reported to be pathogenic to human being and are classified according to their habitats e.g. anthropophiles (from human sources), zoophiles (from animal sources, cats, dogs, cattle, horses, birds or other animals) or geophiles (from soil sources). ${ }^{1,2}$ Clinically, the different types of dermatophytosis are classified according to body site involvement. ${ }^{3}$ Despite the availability of effective antifungal agents, dermatophytic infections continue to be one of the principal dermatological diseases throughout the world especially in tropical countries like Nepal. latrogenic immune suppression in cases of organ transplantation and immunotherapy of various medical conditions, HIV infection further predispose person for extensive resistant type of dermatophytosis. ${ }^{4}$

The need for species identification of dermatophytes in clinical settings is of epidemiological concerns. Infection 
may have animal carriers or linked to recurrent institutional or family outbreaks, may reflect exposure during travel or residence in the area of endemicity or contact with a person with such disease thus it is important for infection control and public health issues as well. ${ }^{1}$

Although dermatophytic infection can be diagnosed by clinical presentation and fungus can be demonstrated by easy laboratory procedures like direct microscopy ( $\mathrm{KOH}$ mount). Fungal species responsible for the infection can be identified by colony characteristic and pigment production. Currently, Fungus responsible for the infection can be identified with great precision by modern technologies like- PCR and Nucleic acid based tests. ${ }^{5,6}$

Present hospital based observational study was carried out to find out the prevalence of dermatophytosis, its clinical presentation and species of fungi responsible for the disease in Central Nepal

\section{METHODS}

It is a prospective observational analysis of study. Clinically suspected 200 cases of dermatophytic infection were included in our study between September 2008 to February 2010. Study was conducted at Department of Dermatology of College of Medical Sciences and Teaching Hospital, Bharatpur, Chitwan. Nepal.

Patients were clinically examined and patient's data were recorded on performa with particular reference to onset of disease, duration and site of involvement, type of initial lesion and progression, associated dermatosis and systemic illness in past and familial occurrence of similar disease. Patients on topical and systemic antifungal treatments were excluded. Screening for ELISA for HIV infection was performed in extensive tinea infection. Skin scraping, hair and nail samples were collected according to standard protocol. In cases of dermatophytosis involving hair bearing areas of body, hair sample was taken by removing hair with intact roots. Nail clipping were taken with sterilised scissor and nail clipper. ${ }^{7}$

Direct microscopy of the samples was performed at the department of Microbiology. The Skin and hair sample were kept in $\mathrm{KOH}$ for 30 minutes and examined for the presence of fungal hyphae and spores but nail clippings were kept for longer time. Samples for culture were collected under sterile precautions and transported to the Department of Microbiology for culture and species identification. Skin samples were inoculated on same day on selective medium with antibiotic. Sabouraud's cyclohexamide chloamphenicol Agar, (HiMedia Laboratories Pvt. Ltd, Mumbai, India,) was used in this study. Reverse of the Petri plates were observed daily for growth, morphology, color, colony texture and pigmentation. If no growth was observed at the end of three weeks, culture was labeled as "negative". Lectophenol blue mounts were prepared for the identification of the structure and morphology of the spores. Clinical photograph of representative skin lesions and colony characteristics of fungus species identified were taken. Microscopic picture of lectophenol blue preparation of fungal culture were taken for the identification of species.The dat were entered in Microsoft Excel 2007 ans statistical analysis was done with SPSS v.16.

\section{RESULTS}

Maximum numbers of cases with dermatophytosis were observed between June to September and almost half of the cases were farmer by occupation. Majority of patients reported for the treatment 5-8 weeks after onset of disease. Dermatophytosis was common in male (56.5\%) as compared to female (43.3\%). The youngest patient was 27 days old and oldest was 77 years old and (40.5\%) of cases were between 26-30 years of age (Fig 1) Most common clinical type observed in our study was Tinea corporis (47\%) followed by T. capitis (12.5\%), T. cruris (12\%), T. pedis (6.5\%), T. unguium (6\%) and T. manum (3\%) respectively. Tinea corporis and T.pedis were higher in female compared with male (M: F; 0.88: 1 ) and (M: $F, 0.6: 1)$ respectively. The patients with concurrent two clinical types were $10 \%$ and two cases with extensive tinea infection were also observed (Fig 3).

Table 1. Results of direct microscope and culture in relation to clinical types of Tinea

\begin{tabular}{llll}
$\begin{array}{l}\text { Clinical } \\
\text { types }\end{array}$ & No. of cases & $\begin{array}{c}\text { Direct microscopy and culture positivity } \\
\text { Direct microscopy } \\
\text { + ve (\%) }\end{array}$ & $\begin{array}{c}\text { Culture } \\
+ \text { ve (\%) }\end{array}$ \\
\hline T. corporis & 94 & $74(78.7)$ & $54(57.4) 2$ \\
\hline T. capitis & 25 & $20(80)$ & $19(76)$ \\
\hline T. cruris & 24 & $15(62.5)$ & $15(62.5)$ \\
\hline $\begin{array}{l}\text { T. unguium } \\
\text { T. pedis }\end{array}$ & 12 & $7(58.3)$ & $8(66.6)$ \\
\hline $\begin{array}{l}\text { T. manum } \\
\text { T. faciale }\end{array}$ & 6 & $6(46.15)$ & $8(61.5)$ \\
\hline $\begin{array}{l}\text { T. corporis/ } \\
\text { cruris }\end{array}$ & 15 & $4(66.6)$ & $4(66.6)$ \\
\hline $\begin{array}{l}\text { T. corporis/ } \\
\text { barbe }\end{array}$ & 1 & $1(25)$ & $3(75.1)$ \\
\hline $\begin{array}{l}\text { T. capitis/ } \\
\text { corporis }\end{array}$ & 2 & $9(60)$ & $7(46.6)$ \\
\hline $\begin{array}{l}\text { T. pedis/ } \\
\text { corporis }\end{array}$ & 1 & $1(100)$ & $1(100)$ \\
\hline $\begin{array}{l}\text { T.unguium/ } \\
\text { corporis/ } \\
\text { cruris }\end{array}$ & 1 & $2(100)$ & $2(100)$ \\
\hline $\begin{array}{l}\text { Extensive } \\
\text { tinea }\end{array}$ & 2 & $1(50)$ & - \\
\hline & & & $1(100)$ \\
\hline
\end{tabular}

Direct microscopic examination $(\mathrm{KOH})$ of the samples reveled overall $71.5 \%$ positive for fungus while $62 \%$ cases were culture positive. High positivity was found in cases of Tinea capitis $80 \%$ followed by Tinea corporis $76.5 \%$ by direct microscopy. Whereas low positivity was observed in Tinea unguium and Tinea pedis $58.3 \%$ and $46.8 \%$ respectively. 
(Table 1) The predominant species of dermatophyte isolated was Trichophyton verrucosum $30.6 \%$ followed by T. rubrum $25 \%$, T. tonsurans $25 \%$ and T. mentagrophytes $8.8 \%$. (Fig 2)T. tonsurans $48 \%$ was found to be predominant species in T. capitis cases. T. rubrum was predominant species in tinea cruris (41.6\%) and $8.3 \%$ in Tinea unguium respectively.

\section{DISCUSSION}

Identification of fungal agent and species responsible for the dermatophytosis is of importance not for the epidemiology but also for therapeutic point of view when treatment is advice for long time. Central Nepal bears tropical climate and maximum number of cases were observed in the month of June to September, when atmospheric temperature and humidity conducive for tinea infection. ${ }^{1,8}$ Agriculture is the dominant profession of the patients $(41.5 \%)$, which includes inhabitant with domestic animals like buffaloes, cows and goats, may leads to transmission of Geo and Zoophilic species of dermatophyte to human beings as over all predominant species of dermatophyte isolated in our study was T. verrucosum, which is normally a zoophilic. ${ }^{9,10}$ In present, study $51 \%$ of patients reported for the treatment after 5 to 8 weeks of onset of disease. This delay in treatment may be because of common practice among local population to take medication over the counter and use of indigenous medications leading to delay in treatment. This may be important cause of interfamilial cases and spread of infection in society.

Overall male predominance was observed (56.5\%) as compared to female $43.3 \%$ in patients with dermatophytosis which is in consistent with various studies. ${ }^{11,12}$ Higher prevalence of Tinea infection in male populations may be because of hard physical labor and wearing shoes for long time predispose them for fungal infection. The commonest age group of Tinea infection reported is between 11 to 20 years of age, but our study showed $40.5 \%$ patients had tinea infections in age group between 26 to 30 years and the youngest patient was 27 days and oldest was 77 years of age. ${ }^{11}$

Preponderance of tinea corporis among female as compared to male (M: F; $0.88: 1)$ in our study is not in accordance with other studies reported. Increase incidence of tinea corporis in females may be because women in Nepal are engaged in farming and other physical labor as a part time work. Physical exercise, working in hot and humid environment increase sweating that may facilitate parasitization of fungus. ${ }^{8,11}$ The most common clinical type observed was Tinea corporis (47\%) followed by Tinea capitis which is in accordance with previous reports in literature. ${ }^{12}$

Prevalence of Tinea capitis was $12.5 \%$ of the patients but study conducted at other parts of Nepal reported rather low (4.6\%) prevalence of tinea capitis whereas prevalence of $15.1 \%$ was reported from Saudi Arabia. ${ }^{13,14}$ The reasons for increase incidence of tinea capitis in this study compare to study from Nepal was because our patients were from boarding school where chances of spread of infection are high. Local custom of wearing caps and scarf, which may facilitate the transmission of infection, could be the reason for increase incidence of tinea capitis in Saudi Arabia. Study carried out in India, noted low prevalence of Tinea capitis and was attributed to the use of mustard oil over scalp and oil reported to have fungistatic property. However, similar study showed no significant association with the use of mustard hair oils in tinea capitis cases. ${ }^{11,12}$ Male predominance in Tinea cruris as compared to female was reported. In agreement with this, male predominance was also observed in our study. Although, Tinea cruris is not common in female but $20.8 \%$ of female cases were also found to have Tinea cruris in our study. ${ }^{3}$ Tinea pedis was higher in age groups between 26 to 30 years with female predominance in our study may be because of agriculture based occupation although male predominance was reported. ${ }^{15}$

The present study noticed male predominance with higher incidence of tinea unguium compare to previous study. This increase incidence in Tinea unguium may be because majority of people are farmer by occupation and there are more chances of transmission of Geo and Zoophilic species. ${ }^{16}$ In present study, an increase in prevalence of Tinea manum was expected as most of the patients were farmer but only $3 \%$ (6 out of 200 cases) had tinea manum. This could be because none of the patients had predisposing factors like anatomical deformities, poor peripheral circulation and pre existing keratoderma. ${ }^{16}$

Study conducted by Aggarwal et al. noted $32 \%$ of patients had two or more clinical types but only $10 \%$ of the patients had concurrently two clinical types in our study. ${ }^{17}$ Tinea corporis with cruris was found to be in $7.5 \%$ cases whereas T. corporis with T. barbae, T. capitis with corporis, T. pedis with T. corporis and Tinea unguium, tinea corporis with cruris were $0.5 \%, 1.0 \%, 0.5 \%$ and $0.5 \%$ respectively. None of the patent with extensive tinea infection was associated with any risk factors and screening ELISA test for HIV infection was negative.On direct microscopic examination $(\mathrm{KOH})$ of sample, Overall $71.5 \%$ of cases were positive for fungus in contrast with studies reported in past. ${ }^{11,12} \mathrm{~A}$ high positivity in Tinea capitis ( $80 \% 0$ was found followed by tinea corporis (76.5\%) Whereas low positivity was observed in cases of Tinea unguium and Tinea pedis $58.3 \%$ and $46.8 \%$ respectively. The possible region for low positivity in Tinea unguium is because of nail specimen takes long time to dissolve and fungal element may not release. However, present study showed high positivity by culture (66\%) as compared to direct microscopy (58.3\%) in Tinea unguium cases. Overall culture positivity was observed in $62 \%$ of cases compare to other studies reported. ${ }^{11,12}$ High positivity in our study could be due to use of selective media, which does not, allowed contaminants to grow.

T. rubrum is the predominant species reported worldwide, but epidermaophyton also reported as a dominant species, 
In contrast with their findings, present study showed Trichophyton verrucosum (30.6\%) as a predominant species followed by T. rubrum (25\%).\%) . Moh. Reza et al. also documented T. verrucosum as a dominant species in their study. ${ }^{10,18,19}$

In Tinea capitis, T. tonsurans (48\%) was found to be predominant species followed by T. violaceum (20\%) but $\mathrm{T}$. violaceum as the main causative agent of tinea capitis reported from other part of Nepal. ${ }^{13}$ Although E. floccosum is reported to be causative organism of $\mathrm{T}$ cruris but $\mathrm{T}$. rubrum $41.6 \%$ was isolated from Tinea cruris so do in cases of $\mathrm{T}$ unguium and which is in accordance to the various reports in literature. Isolation of T. mentagrophytes in Tinea faciae case suggests infection with anthropophilic fungus from household contact. ${ }^{14}$ In patients with extensive tinea infection (T. corporis, cruris, manum, unguium and pedis) T. verrucosum was isolated from all the sites that further suggest infection with Zoophilic.

\section{CONCLUSION}

The study highlighted Tinea corporis as the most common clinical pattern of dermatophytosis with female predominance followed by Tinea capitis in Central Nepal. Overall predominant causative fungal species isolated was Trichophyton verrucosum followed by T. rubrum and T. tonsurans. Because of the inhabitant of house hold animals, zoophilic fungi become the dominant pathogen of human beings instead of Anthropophilic thus Fungal species may varies from place to place and even at same continent time to time. Fugal cultures are mandatory specially in cases of T.unguim to improve the diagnosis when prolong treatment is required.

\section{REFERENCES}

1. Havlickova B. Epidemiological treands in skin mycoses worldwide, Mycoses, 2008;51:2-15.

2. Ajello L. Present day concepts in the dermatophytes.Mycopatho. Myco.Appl. 1962; 17:315-24.

3. Hay RJ, Moore MK. Dermatophytosis. In: Tony Burns, Stephen Breathnach, Neil Cox and Christopher Griffiths edi, Rook's Text book of Dermatology 7th ed., Blackwell Science Ltd, Blackwell Publishing company UK; 2004: 31.1-74.

4. Tossander J, Karlsson A, Morfeldt-Mason L et al. Dermatophytosis and HIV infection: a study in Homosexual men. Acta Derm venerol 1988:68:53-9.

5. Rippon J.W Dermatophytoses and Dermatomycosis in: Medical Mycology, the pathogenic fungi and pathogenic actinomycetes, 2nd ed.W.B. Saunders Co; Philadelphia; 1982:154-248

6. M. Arbatzis, Diagnosis of Common dermatophyte infections by novel multiplex real time polymerase chain reaction/identification scheme. British journal of Dermatology. 2007; 157:681-89

7. Chandra J. A text book of medical mycology 3rd edition. New Delhi: Mehta Publishers; 2009: 91-113.

8. Philpot $\mathrm{CM}$. Some aspects on the epidemiology of tinea. Mycopathologia 1997; 3: 62.

9. Mary MM. Human cases of cattle ringworm due to Trichophyton verrucosum in Victoria, Australia. Australasian Journal of Dermatology. 2001; 41( 2): 90-4.

10. Mohammad RA, Seyed AG. Dermatophytes as a cause of epizoonoses in dairy cattle and humans in Iran: Epidemiological and clinical aspects of mycosis early view. 23 Dec 2009; 41: 90-4.

11. Bindu V. Clinico - mycological study of dermatophytosis in Calicut, Indian J Dermatol Venereol Leorol 2002; 68: 259-61.

12. Singh S, Beena PM. Profile of dermatophyte infections in Baroda Indian J Dermatol Venereol Leorol 2003; 69: 281-3.

13. Jha BN, Garg VK, Agrawal S. Tinea Capitis in Eastern Nepal. International Journal of Dermatology 2006; 45(2): 100-2.

14. Abanmi A, Bakheshwaim.S, EL Khizzi N. Characteristics of superficial fungal infections in the Riyadh region of Saudi Arabia. Int J Dermatol. March 2008; 47:229-35.

15. S. Lupa, Seneczko F, Jeske JG. Epidemiology of dermatomycoses of human in central Poland, Part III: Tinea pedis. Mycoses 1999; 42: 5635.

16. Perea S, Ramos MJ, Garau M. Prevalence and Risk Factors of Tinea Unguium and T. Pedis in General population in Spain. Journal of clinical Microbiology 2000; 3:3226-30.

17. Agrawalla A, Jacob M, Sethi M. A clinico mycological study of Dermatophytoses in Nepal. Journal of dermatology.2001; 28:16-21.

18. Tietz HJ, Kunzelmann V, Scoenian G. Changes in the fungal spectrum of dermatophytosis. Mycoses 1995; 38(S.1); 33-9.

19. Shahindokht B, Ali AK. Epidemiological survey of dermatophytosis in Tehran, Iran from 2000 to 2005. Indian JDermatol Venereol leprol March 2009;75:142. 